\title{
Development of ultrafast broadband electronic sum frequency generation for charge dynamics at surfaces and interfaces
}

Cite as: J. Chem. Phys. 150, 024708 (2019); https://doi.org/10.1063/1.5063458

Submitted: 27 September 2018 . Accepted: 20 December 2018 . Published Online: 14 January 2019

Gang-Hua Deng, Yuqin Qian, and Yi Rao (iD)
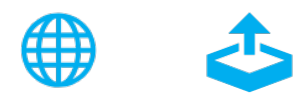

\section{ARTICLES YOU MAY BE INTERESTED IN}

Enhancing the signal strength of surface sensitive 2D IR spectroscopy

The Journal of Chemical Physics 150, 024707 (2019); https://doi.org/10.1063/1.5065511

Broadband models and their consequences on line shape analysis in vibrational sumfrequency spectroscopy

The Journal of Chemical Physics 149, 174703 (2018); https://doi.org/10.1063/1.5053128

Water structure at the interface of alcohol monolayers as determined by molecular dynamics simulations and computational vibrational sum-frequency generation spectroscopy

The Journal of Chemical Physics 150, 034701 (2019); https://doi.org/10.1063/1.5072754

PHYSICS TODAY WHITEPAPERS
ADVANCED LIGHT CURE ADHESIVES

Take a closer look at what these

environmentally friendly adhesive systems can do
READ NOW

PRESENTED BY 8. MASTERBOND* 


\title{
Development of ultrafast broadband electronic sum frequency generation for charge dynamics at surfaces and interfaces
}

\author{
Cite as: J. Chem. Phys. 150, 024708 (2019); doi: 10.1063/1.5063458 \\ Submitted: 27 September 2018 - Accepted: 20 December 2018 • \\ Published Online: 14 January 2019
}

Gang-Hua Deng, Yuqin Qian, and Yi Rao

\section{AFFILIATIONS}

Department of Chemistry and Biochemistry, Utah State University, Logan, Utah 84322, USA

Note: This article is part of the Special Topic "Nonlinear Spectroscopy and Interfacial Structure and Dynamics" in J. Chem. Phys. a) Electronic mail: yi.rao@usu.edu

\begin{abstract}
Understandings of population and relaxation of charges at surfaces and interfaces are essential to improve charge collection efficiency for energy conversion, catalysis, and photosynthesis. Existing time-resolved surface and interface tools are limited to either under ultrahigh vacuum or in a narrow wavelength region with the loss of spectral information. There lacks an efficient time-resolved surface/interface-specific electronic spectroscopy under ambient conditions for the ultrafast surface/interface dynamics. Here we developed a novel technique for surface/interface-specific broadband electronic sum frequency generation (ESFG). The broadband ESFG was based on a stable two-stage $\mathrm{BiB}_{3} \mathrm{O}_{6}$ crystal-based optical parametric amplifier, which generates a strong broadband short-wave infrared (SWIR) from $1200 \mathrm{~nm}$ to $2400 \mathrm{~nm}$. A resultant surface spectrum covers almost all visible light from $480 \mathrm{~nm}$ to $760 \mathrm{~nm}$, combined a broadband electronic second harmonic generation (ESHG) with the ESFG from the SWIR laser source. We further developed the steady-state and transient broadband ESFG and ESHG techniques to investigate the structure and dynamics of charges at oxidized p-type GaAs (100) semiconductor surfaces, as an example. Both the steady-state and transient experiments have shown that two surface states exist inside the bandgap of the GaAs. The kinetic processes at the GaAs surface include both the population and recombination of the surface states after photoexcitation, in addition to the build-up of the space photo-voltage (SPV). The build-up SPV occurs with a rate of $0.56 \pm 0.07 \mathrm{ps}^{-1}$, while the population rate of the surface states exhibits a two-body behavior with a rate constant of $(0.012 \pm 0.002) \times 10^{12} \mathrm{~s}^{-1} \mathrm{~cm}^{2}$. The photo-generated electron-hole pairs near the surface recombine with a rate of $0.002 \pm 0.0002 \mathrm{ps}^{-1}$ for the oxidized p-type GaAs (100). All the methodologies developed here are readily applied to any optically accessible interfaces and surfaces, in particular buried interfaces under ambient conditions.
\end{abstract}

Published under license by AIP Publishing. https://oi.org/1 0.1063/1.5063458

\section{INTRODUCTION}

Surface/interfacial states are donor states or acceptor states and act as mediators for trapping and recombination of charge carriers in semiconductors. ${ }^{1-4}$ The knowledge of surface/interfacial states and, particularly, their energy levels and relaxation processes is critical for understanding the fundamental mechanisms of inorganic and organic semiconductors in the process of energy conversion, catalysis, and photosynthesis. For example, on a semiconductor surface, the excess charge in surface states is balanced by bulk charge within a small space-charge region (SCR) on the order of a few nanometers, leading to band bending near the surface. ${ }^{1,2,5,6}$ After photo-excitation, charge separation of generated electron-hole pairs in the strong electric field of the SCR is responsible for band flattening. ${ }^{7}$ These surface charge properties dominate the optical and electrical characteristics of semiconductor materials. On the other hand, the determination of energy levels for surface states allows for identifying which mechanism governs the Fermi energy at surfaces such 
as intrinsic surface state pinning, surface defect pinning, and bulk doping for unpinned surfaces. Although there are many surface tools for the determination of surface states, ${ }^{1,8-12}$ there lacks an efficient surface/interface-specific electronic spectroscopy under ambient conditions for ultrafast dynamics of inorganic and organic semiconductors occurred at surfaces and interfaces.

Recent studies showed that charge transfer occurs at semiconductor/organic thin film interfaces with timeresolved electric-field induced second harmonic generation (SHG), with the loss of spectral information. ${ }^{13-16}$ Early efforts have also demonstrated the ultrafast relaxation of the surface photo-voltage (SPV) effect on semiconductors by timeresolved photoemission spectroscopy. ${ }^{17-19}$ The time evolution of the electric field was demonstrated in the surface layer of semiconductors by measuring the kinetic-energy shifts of photoelectrons. ${ }^{20}$ However, these experiments could not detect real surface properties since the methods used were not surface-specific. Sum frequency generation (SFG) and second harmonic generation (SHG) have been proven to be versatile nonlinear optical spectroscopic tools for investigations of structures, kinetics, dynamics, and chemical reactions occurred at surfaces and interfaces. ${ }^{21-61}$ SFG is the second order process generated by two fundamental beams, in which one is fixed and the other is tunable or broadband. In fact, SHG is a special case of SFG when the two incident beams are degenerate. Thanks to recent advances in solid-state lasers, the SFG technique continues to evolve into an analytical tool for applications relevant to environment, catalysis, materials, and biology. As a surface/interfacespecific tool, SFG is anticipated to provide vibrational structures as well as electronic features, both of which are essential in understanding chemical and physical properties occurred at interfaces. Previous attempts were mainly concentrated on vibrational SFG (VSFG) experiments. ${ }^{22,26}$ In particular, recent efforts in a femtosecond broadband IR source have revolutionized VSFG experiments in terms of both ultrafast time scale $30,39,54,62-66$ and high-resolution spectrum. ${ }^{51,67}$ On the other hand, the development of electronic sum frequency generation (ESFG) is much left behind. The main reason for this lies in the fact that there lacks a broadband laser source with stable and high output energy for ESFG experiments.

Historically, ESFG experiments were implemented by detecting SHG and point-by-point tuning laser wavelength. ${ }^{68-73}$ Such experiments are considered as tuning SHG experiments, relying on dye lasers, or tunable Ti:sapphire oscillators or optical parametric oscillators (OPO), or optical parametric amplifiers (OPAs). The tuning SHG experiments are time-consuming. Moreover, wavelength calibrations and intensity normalization are challenging in that the divergence and pointing from the dye lasers, OPO, or OPA vary greatly with angular crystal tuning and different dyes. In recent years, tremendous efforts have been made in broadband ESFG experiments. ${ }^{38,71,74}$ These pioneering studies initiated a lot of interest from the community of interfacial nonlinear optical spectroscopies. In their experiments, the authors introduced a multi-filament white light continuum by focusing a strong femtosecond laser onto flowing water. ${ }^{38,74}$ Analogous to the broadband VSFG, a broadband visible source was used to implement electronic SFG. However, the chirp with the white light was a serious problem in the ESFG experiments. Both one-photon and two-photon resonant signals also complicate the interpretation of electronic spectra from these experiments. As a consequence, the temporal chirp and the spectral complexity in the ESFG probe could prevent transient ESFG spectroscopy from revealing ultrafast structural dynamics and chemical reactions at surfaces and interfaces.

In this work, we presented a novel ESFG spectroscopy for probing ultrafast electronic properties at surfaces and interfaces. The approach relies on the construction of a stable and high power optical parametric amplifier (OPA) based on large aperture bismuth triborate, $\mathrm{BiB}_{3} \mathrm{O}_{6}$ (BIBO) crystals, pumped at $1 \mathrm{kHz}$ by the fundamental of a Ti:sapphire amplifier. Here, we first demonstrated the build-up of a BIBO-based OPA with stable and high output power. With the broadband OPA, we then developed both steady-state and transient broadband ESFG and electronic second harmonic generation (ESHG) experiments for semiconductor surfaces. We took the oxidized p-type GaAs (100) surface as an example to demonstrate the ultrafast broadband ESFG spectroscopy.

\section{EXPERIMENTAL SECTION}

\section{A. Construction of a broadband short-wave IR (SWIR) OPA}

The concept of a broadband short-wave IR amplifier is based upon an OPA that under achromatic phase-matching conditions, zero group velocity mismatch (GVM) is satisfied between the idler $\left(\lambda_{\mathrm{I}}\right)$ and signal $\left(\lambda_{\mathrm{S}}\right)$ pulses for collinear interaction at $1600 \mathrm{~nm}$. In the case of type-I collinear interaction, this condition can be fulfilled near degeneracy $\left(\lambda_{\mathrm{S}} \approx \lambda_{\mathrm{I}}\right)$. However, the bandwidth can be further improved by the second condition that the group velocity dispersion (GVD) of the signal and idler pulses disappears. For birefringent nonlinear crystals like Beta Barium Borate (BBO), the second condition is simultaneously satisfied only at $\lambda_{\mathrm{P}}$ far from $800 \mathrm{~nm}$. On the other hand, for the biaxial BIBO crystal, the second condition is readily satisfied near $800 \mathrm{~nm}$ that is available from a commercial femtosecond amplifier laser. The optical parametric interaction in the $x-z$ principal plane of the BIBO unites broad spectral feature, high effective nonlinearity, and small GVM between the amplified signal and idler, and the pump pulses. ${ }^{75}$ $\mathrm{BIBO}$ is a relatively new nonlinear optical crystal and has effective second-order nonlinearity $d_{\text {eff. }}$. It possesses large angular and spectral acceptance bandwidths, highly efficient phasematching properties, small spatial walk-off, and broadband angle tuning at room temperature. BIBO also has broad optical transparency extending from $\sim 280 \mathrm{~nm}$ in the UV up to $\sim 2.7 \mu \mathrm{m}$ in the infrared. ${ }^{75-77}$ These features make the BIBO crystal an ideal choice for down-conversion of amplified femtosecond 
pulses from a commercially available $800 \mathrm{~nm}$ solid-state laser without two-photon absorption.

In our work, a two-stage OPA was designed to generate a broadband short-wave infrared (SWIR) from $1200 \mathrm{~nm}$ to $2400 \mathrm{~nm}$. The ultrabroadband SWIR is the combination of both signal (1200-1600 nm) and idler (1600-2400 nm). Experimentally, a fundamental light with a center wavelength of $796 \mathrm{~nm}$ and a pulse duration of 100 fs was generated by a $1 \mathrm{kHz}$ Ti-sapphire amplified laser system (UpteK Solutions). A portion of $1.5 \mathrm{~mJ}$ was used for the broadband OPA. Figure 1(a) shows a schematic of our home-built broadband OPA. A collinear geometry was adopted in order for the broadband SWIR to be free of spatial walk-off. The broadband OPA consists of the generation of a white light, the first-stage amplifier, and the second-stage amplifier. The non-trivial part for the broadband OPA is the generation of a white light continuum as a regular OPA. We used a 3-mm thick YAG crystal to generate a white light continuum instead of a sapphire plate reported in the literature. ${ }^{75}$ A pinhole was placed in front of the focal length in the light path of the white light generation for fine adjustments. Together with a tunable neutral density filter, a stable single filament white light was produced. Uncoated $3 \mathrm{~mm}$ thick BIBO crystals were cut at $\theta=11.4^{\circ}$ (Eksma) for both the first-stage amplifier and the second-stage amplifier. With an input energy of $200 \mu \mathrm{J}$, the output of an amplified first stage was expected to be $2-4 \mu \mathrm{J}$ with a fluence of less than $80 \mathrm{GW} / \mathrm{cm}^{2}$. A broadband spectrum from the first-stage amplifier could be readily detected by a SFG from a z-cut quartz single crystal surface with a mixing of a picosecond $796 \mathrm{~nm}$. For the second-stage amplifier with an input of

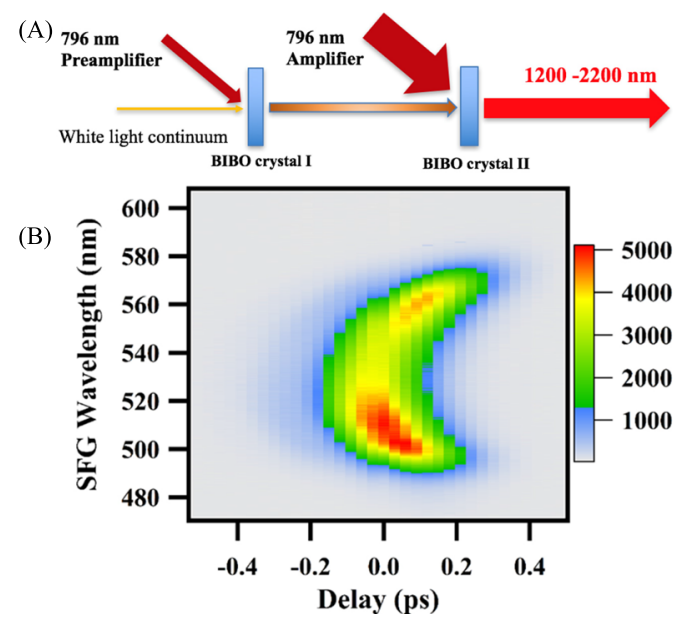

FIG. 1. (a) A schematic of a broadband BIBO-based OPA. The OPA consists of two-stage amplifiers. A white light continuum mixes on a BIBO crystal with a small portion of a $796 \mathrm{~nm}$ pump for a preamplifier, followed by further amplification on the second BIBO crystal with a large portion of a $796 \mathrm{~nm}$ pump. (b) Interfacial XFROG from a z-cut quartz single crystal surface by mixing a femtosecond $796 \mathrm{~nm}$ with the broadband and short laser sources. The short-wave IR wavelength was found to cover from $1200 \mathrm{~nm}$ to $2200 \mathrm{~nm}$. The pulse duration of the output beam was on the order of $200 \mathrm{fs}$.
$1.2 \mathrm{~mJ}$, a pump fluence was $60 \mathrm{GW} / \mathrm{cm}^{2}$. Seeding with the signal pulse from the first-stage, a pulse energy of $250 \mu \mathrm{J}$ was readily obtained from the second-stage (the signal plus the idler). It can be possible to obtain a higher output energy for broadband OPA by using a 5-mm thick BIBO crystal.

Generally, it is challenging to characterize temporal and spectral properties from the ultra-broadband SWIR. The main challenge is to find an ultrathin nonlinear optical single crystal for cross frequency-resolved optical grating (XFROG). To circumvent the difficulty, we measured frequency-resolved optical gating with interfacial reflection SFG responses (Interfacial XFROG) from a z-cut quartz single crystal (MTI Corporation). The interfacial SFG process satisfies the phase matching condition with a coherent length as short as 10-20 nm (see the supplementary material). Figure 1(b) shows interfacial XFROG from a z-cut quartz single crystal by mixing a femtosecond $796 \mathrm{~nm}$ with the broadband and short laser sources. The wavelength for the broadband SWIR was obtained from the SFG wavelength by considering energy conservation of the $796 \mathrm{~nm}$ and the broadband SWIR. The SWIR wavelength was found to cover from $1200 \mathrm{~nm}$ to $2400 \mathrm{~nm}$ from Fig. 1(b). The efficiency from $2200 \mathrm{~nm}$ to $2400 \mathrm{~nm}$ is slightly low probably due to a poor reflection for the wavelength range from the dichroic mirror after the second BIBO in our setup. This laser output is extended down to $600 \mathrm{~nm}$ by using a $0.2 \mathrm{~mm}$ BBO crystal. It is seen from the frequency-resolved SFG measurements that the broadband SWIR exhibited a pulse duration of $200 \mathrm{fs}$. These ultra-broadband and short pulse durations of the SWIR make the laser source a good choice for interfacial electronic sum frequency generation experiments.

\section{B. Steady-state broadband ESFG and ESHG experiments}

Figure 2 shows a schematic setup for transient broadband ESFG and ESHG apparatus. To implement ESFG experiments, a picosecond $796 \mathrm{~nm}$ light was produced by a homebuilt $4-f$ configuration pulse shaper. ${ }^{43,78,79}$ It generally consists of a cylindrical lens (focal length $=25 \mathrm{~cm}$ ), a grating (1200 grooves $/ \mathrm{mm}$ ), adjustable slits, and several highly reflective mirrors. The pulse duration and spectral resolution are readily varied by controlling the width of the slit. We obtained an bandwidth of $15 \mathrm{~cm}^{-1}$ with 2 ps pulse duration. The input energy of $2.0 \mathrm{~mJ}$ fundamental light generates $60 \mu \mathrm{J}$ output of the picosecond laser. A one-inch $\mathrm{CaF}_{2}$ lens with $150 \mathrm{~mm}$ focus length was applied to focus the SWIR fundamental light onto the sample at an angle of $45^{\circ}$ to the normal of the sample. The incident angle of picosecond $800 \mathrm{~nm}$ was set at $60^{\circ}$. A broadband polarizer was used to choose the polarization of the fundamental light. It is noted that the fundamental broadband SWIR light first went through a series of filters, including one $0.25 \mathrm{~mm} \mathrm{Si}$ wafer (MTI Corp.), one $1600 \mathrm{~nm}$ short-pass filter (Edmund, only used for ESHG experiments to avoid third harmonic generation in the range of 600-760 nm), and one $1000 \mathrm{~nm}$ long-pass filter (Reynard) to remove residual $796 \mathrm{~nm}$ light and high-order harmonic light generated by the optics. Both the ESFG and ESHG signals were measured 


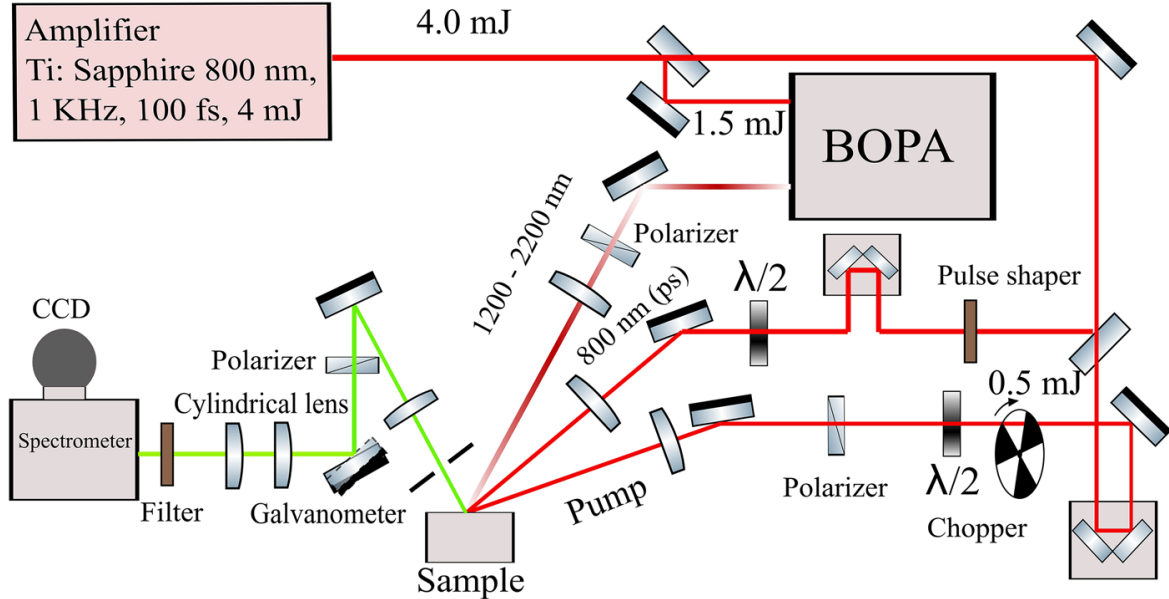

FIG. 2. A schematic of transient broadband ESFG and ESHG apparatus. A fundamental light with a center wavelength of $796 \mathrm{~nm}$ and a pulse duration of $100 \mathrm{fs}$ was generated by a $1 \mathrm{kHz}$ Ti-sapphire amplified laser system. One portion of $1.5 \mathrm{~mJ}$ was used for the broadband OPA The second portion of $2.0 \mathrm{~mJ}$ fundamental light was used to generate the $60 \mu \mathrm{J}$ output of the picosecond laser with a home-built pulse shaper. The remaining of $0.5 \mathrm{~mJ}$ was used as a pump beam. A single-axis scanning Galvo mirror was applied by rotating the mirror with a small angle difference between them to separate the ESFG/ESHG signal with pump-on and pump-off at a frequency of $500 \mathrm{~Hz}$.

by a combination of a spectrometer (Andor-2300i, Princeton Instrument) with a liquid nitrogen-cooled CCD (Princeton Instrument, back-illuminated $1300 \times 400)$. A Winspec software provided by Princeton Instrument or a self-complied Labview program was used to implement the data acquisition for steady-state ESFG and ESHG signals. For the broadband ESHG, we simply blocked the picosecond $796 \mathrm{~nm}$ and used only SWIR with a $1600 \mathrm{~nm}$ short-pass filter to carry out experiments. The SHG light beam is directional and goes along the reflected broadband SWIR by satisfying energy and momentum conservations. To measure the ESHG signal, the light along the reflection direction of the fundamental light was collected that includes both the fundamental light and ESHG signal. Subsequently, the fundamental light was filtered out combining three optical filters, including a $945 \mathrm{~nm}$ short-pass filter (Semrock), a $600 \mathrm{~nm}$ long-pass filter (Reynard), and a $785 \mathrm{~nm}$ short-pass filter (Semrock).

The intensity for ESFG and ESHG is given by $21,24,69$

$$
\mathrm{I}\left(\omega_{\mathrm{SF} / \mathrm{SH}}\right) \propto\left|\chi^{(2)}\left(\omega_{\mathrm{SF} / \mathrm{SH}}\right)\right|^{2}=\left|\frac{\mathrm{N}_{\mathrm{s}}\left\langle\beta^{(2)}\right\rangle}{\omega-\omega_{i}+i \Gamma}\right|^{2},
$$

where $\chi^{(2)}\left(\omega_{\mathrm{SF} / \mathrm{SH}}\right)$ represents macroscopic susceptibilities from interfaces and surfaces for ESFG or ESHG, $\mathrm{N}_{\mathrm{s}}$ denotes surface density, $\beta^{(2)}$ denotes the molecular hyperpolarizability, $\omega_{i}$ denotes the $i$ th electronic transition frequency, and $\Gamma$ denotes the damping rate of electronic transitions.

\section{Transient broadband ESFG and ESHG experiments}

To investigate photo-excited relaxation processes at surfaces and interfaces, a pump laser was introduced to carry out transient broadband ESFG and ESHG experiments. The fundamental $796 \mathrm{~nm}$ light was used as a pump pulse for p-type GaAs (MTI, $0.5 \mathrm{~mm}$ thick) surfaces. A motorized translational stage (Klinger) was used to control a time delay between the pump and probe pulses. A pair of a half-wave plate (Thorlabs) and a polarizer (Thorlabs) was used to precisely tune the fluence of the pump pulse and to control its polarization. The pump light was further spatially overlapped with the ESFG/ESHG probe by a 1-in. lens with a focal length of $500 \mathrm{~mm}$ at an incident angle of $37^{\circ}$ with respect to the surface normal.

Transient broadband ESFG and ESHG spectra measure a few percent or less change in the probes. This was achieved by setting the working frequency of the optical chopper at $500 \mathrm{~Hz}$ synchronized to the $1 \mathrm{kHz}$ laser system to measure the transient signal with and without the pump. Distinct from a traditional transient absorption method, ESFG and EHG probe signals are generally weak. A fast data acquisition could bring large readout noises in ESFG and ESHG experiments. To separate the ESFG/ESHG signal with pump-on and pump-off, a single-axis scanning Galvo mirror ${ }^{80}$ (Thorlabs) was applied by rotating the mirror up and down with a small angle difference between them, as schematically shown in Fig. 2. As such, the ESFG/ESHG signal was imaged vertically separated into two strips onto a CCD chip by both a vertically focal cylindrical lens of $25 \mathrm{~cm}$ and a horizontally focal cylindrical lens of $10 \mathrm{~cm}$. Since the scanning galvanometer system was synchronized with a chopper frequency of $500 \mathrm{~Hz}$, the resultant two strips on the CCD chip corresponded to the ESFG/ESHG signal with pumpon and pump-off, respectively. A Labview program was compiled to control the acquisition of the ESFG/ESHG signal with pump-on and pump-off, as well as the time delay between the broadband ESFG/ESHG probe and the pump light. The change in transient ESFG/ESHG signal is calculated as ${ }^{54,65,81,82}$

$$
\frac{\Delta I}{I}(\omega, t)=\frac{I_{\text {on }}(\omega, t)-I_{\text {off }}(\omega, t)}{I_{\text {off }}(\omega, t)},
$$

where $I_{o n}(\omega, t)$ and $I_{\text {off }}(\omega, t)$ are the ESFG/ESHG signals with the pump-on and the pump-off, respectively. In the presence of a pump, $I_{\text {on }}$ is proportional to $\left|\chi^{(2)}+\Delta \chi^{(2)}\right|^{2}$, where $\Delta \chi^{(2)}$ is the photoinduced change in surface susceptibility $\chi^{(2)}$. Combining with Eq. (1), rearrangement of Eq. (2) gives $54,65,81,82$

$$
-\frac{\Delta I}{I}(\omega, t)=\frac{2 \Delta \chi^{(2)}}{\chi^{(2)}}
$$


Time-dependent change in ESFG/ESHG, $-\frac{\Delta I}{I}(\omega, t)$, provides time evolution of ground state recovery, photo-induced absorption of new species, other relaxations, and intermolecular interactions occurred at surfaces and interfaces.

\section{RESULTS AND DISCUSSION}

In what follows, we shall demonstrate how we applied the broadband electronic SFG to investigate the structure and dynamics of surface states at surfaces and interfaces. All methodologies developed in these cases are readily applied to any surfaces and interfaces that are optically accessible.

Steady-state ESFG and ESHG measurements were performed on the oxidized p-type GaAs (100) surface. Oxidized p-type (Zn-doped, 1.3-2.2 $\times 10^{19} \mathrm{~cm}^{-3}$ ) GaAs wafers with (100) surface termination were purchased from MTI Corporation. SSP and S-in/P-out polarization combinations were used for the ESFG and ESHG, respectively. SSP for the ESFG represents an S-polarized SFG output beam $\left(\omega_{\mathrm{SF}}\right)$, an S-polarized visible input beam $\left(\omega_{\text {vis }}\right)$, and a P-polarized input SWIR $\left(\omega_{\text {IR }}\right)$. $\mathrm{S}$-in/P-out for the ESHG refers to an S-polarized incident beam $\left(\omega_{1}\right)$ and a p-polarized output SHG beam $\left(\omega_{2}\right)$. A fourfold symmetry at the GaAs surface was observed as reported in the literature in the case of SSP for $\mathrm{SFG}^{83}$ and S-in-P-out for SHG. ${ }^{84-87}$ Bulk contributions were eliminated by setting the azimuthal angle to be $45^{\circ}$ with respect to the direction of GaAs [110]. Azimuthal angle-dependent experiments in our experiments are in good agreement with those reported in the literature. ${ }^{83-87}$

To obtain broad surface electronic spectra, we measured both ESFG and ESHG responses from p-type oxidized GaAs (100). The ESFG spans from $480 \mathrm{~nm}$ to $600 \mathrm{~nm}$ and measures surface second-order responses by mixing the spectrally narrow $800 \mathrm{~nm}$ with the SWIR beam. On the other hand, the ESHG gives a spectral region from $600 \mathrm{~nm}$ to $765 \mathrm{~nm}$ by employing part of the SWIR beam with a short-pass filter cutoff at $1600 \mathrm{~nm}$. Together, the steady-state surface spectra cover almost a majority of the visible region from $480 \mathrm{~nm}$ to $765 \mathrm{~nm}$. This makes our second-order nonlinear electronic spectrometer a very unique tool for studying interfacial electronic responses into applications of materials, environment, and biology. The inset of the top panel in Fig. 3(a) displays the steady-state surface spectra from p-type oxidized GaAs (100). The polarization combinations for ESFG and ESHG were set at SSP and S-in/P-out, separately. The steady-state ESFG and ESHG spectra were normalized to that from a BBO surface. The steady-state surface electronic spectrum from p-type oxidized GaAs (100) shows only a broad peak above $600 \mathrm{~nm}$, which is much larger than its energy gap of $870 \mathrm{~nm}$. The broad peak was attributed to the transition from the surface states near the valence band to a higher valley of the p-type GaAs.

Figure 3(a) presents two-dimensional pseudo-color transient broadband surface spectra of p-type oxidized GaAs (100) under the photoexcitation of $796 \mathrm{~nm}$. The transient spectra from bulk contribution are found in Fig. S1 of the supplementary material. The transient change occurs mainly in the
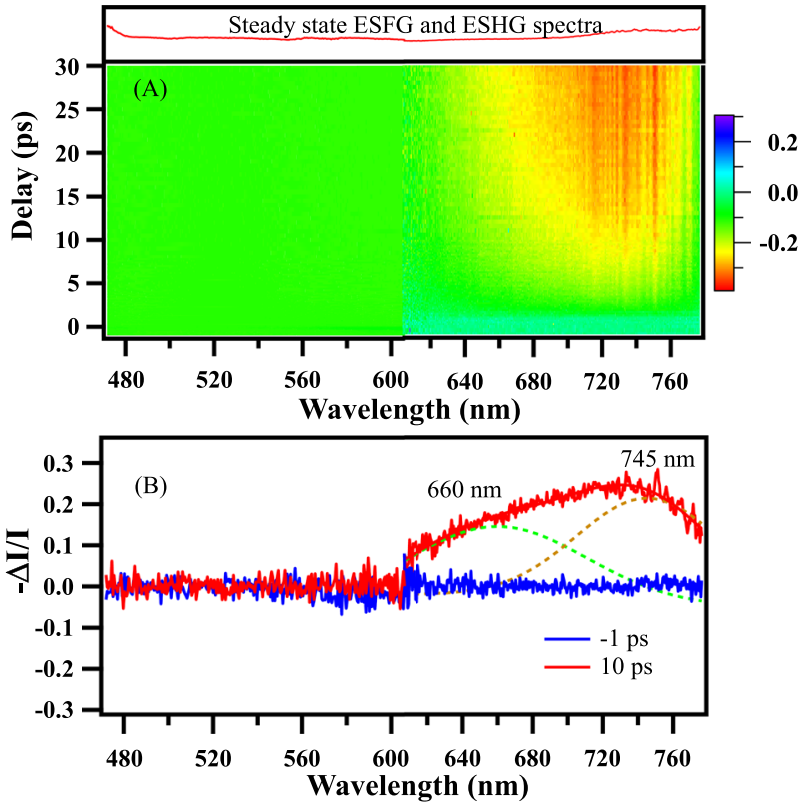

FIG. 3. (a) Two-dimensional pseudo-color transient broadband surface spectra from the p-type oxidized GaAs (100) surface, as compared with steady-state surface spectra in the inset (the red line). (b) The transient spectra for -1 ps and 10 ps taken from the two-dimensional spectra in (a). The transient spectrum at 10 ps was fitted by two Gaussian peaks. One is located at around $660 \mathrm{~nm}$ (green dashed line), and the other is at around $745 \mathrm{~nm}$ (brown dashed line). The pump wavelength was set at $796 \mathrm{~nm}$, and the pump fluence was kept at $1.27 \mu \mathrm{J} / \mathrm{cm}^{2}$

spectral region of $600 \mathrm{~nm}-760 \mathrm{~nm}$. Since the surface susceptibilities of $\chi_{\mathrm{XXZ}}^{(2)}$ and $\chi_{\mathrm{XZX}}^{(2)}$ for GaAs are negative in sign, ${ }^{85}$ the decrease in the transient surface signal corresponds to the increase in number density of new species, namely, the population and relaxation of surface states in the case of GaAs. Two Gaussian peaks are used to fit the transient surface responses in the spectral region of $600 \mathrm{~nm}-765 \mathrm{~nm}$. One is located at around $660 \mathrm{~nm}$, and the other is located at around $745 \mathrm{~nm}$, as seen in Fig. 3(b) for the p-type GaAs (110). The transient spectra at different delays (see Fig. S2 of the supplementary material) also displays no spectral shift. In general, the resonant peaks could arise from either one-photon or two-photon or both. Specifically, the transient signals from $600 \mathrm{~nm}$ to $765 \mathrm{~nm}$ can be resonant with either the fundamental light (1200 nm$1500 \mathrm{~nm}$ ) or the doubling frequency $(600 \mathrm{~nm}-765 \mathrm{~nm})$ in the electronic SHG process. If the ESHG is resonant with the fundamental light ( 1320 nm and $\sim 1490 \mathrm{~nm})$, it was expected to observe two distinct peaks around $497 \mathrm{~nm}$ and $519 \mathrm{~nm}$ by mixing the two fundamental lights with the $796 \mathrm{~nm}$ to conserve the energy conservation in transient ESFG spectra. No any clear features around $497 \mathrm{~nm}$ and $519 \mathrm{~nm}$ were observed in the transient ESFG. This suggests that the two peaks in transient ESHG spectra are due to a two-photon resonance of $\sim 660 \mathrm{~nm}$ and $\sim 745 \mathrm{~nm}$ from the surface states of oxidized p-type GaAs. The resulting spectra were attributed to the resonant transitions from the surface states to a higher valley with the doubling frequency. From these analyses, the two surface states 
are suggested to likely exist in oxidized p-type GaAs. These two surface states in GaAs were also observed in the previous work: ${ }^{88-90}$ a dominant acceptor state located at $E_{\mathrm{as}}=0.75 \mathrm{eV}$ above the valence-band edge and a secondary donor state located at $E_{\mathrm{ds}}=0.50 \mathrm{eV}$. The surface states are not pinned at the mid-gap (Fermi level pinning) in the oxidized p-type GaAs. Our experimental results are in qualitative agreement with those in the literature. ${ }^{88-90}$

To reveal the population and recombination rates of the surface states, we took kinetic profiles in Fig. 3(a) for the two surface states of p-type GaAs (100), as shown in Fig. 4. Timedependent surface signals represent how the surface states are initially populated in GaAs and how the populated surface states subsequently relax over time. It is seen in Fig. 4 that the time-dependent responses at $660 \mathrm{~nm}$ and $745 \mathrm{~nm}$ were found to be almost similar, suggesting that both the surface states possess almost the same capability of trapping surface electrons. The decay trace was readily fitted with a single exponential function, yielding a time constant of $\sim 500 \pm 40 \mathrm{ps}$. This process originates mainly from the surface electron-hole recombination of p-type GaAs. The electron-hole recombination at the surface is much slower than that of $\sim 103 \pm 10 \mathrm{ps}$ in the bulk in Fig. 4. On the other hand, the early kinetic rise for

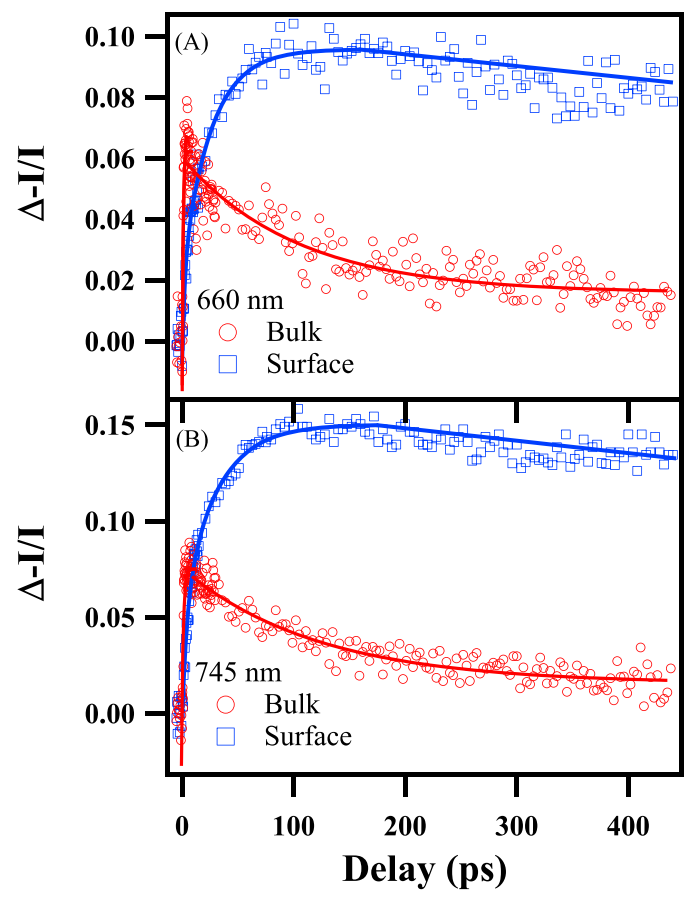

FIG. 4. Comparison of kinetic profiles of the surface and bulk for p-type GaAs (100) at $660 \mathrm{~nm}$ (a) and at $730 \mathrm{~nm}$ (b). The pump fluences for the surface experiments and the bulk experiments were kept at $1.27 \mu \mathrm{J} / \mathrm{cm}^{2}$ and $381 \mu \mathrm{J} / \mathrm{cm}^{2}$, respectively. The rise times for $660 \mathrm{~nm}$ and $745 \mathrm{~nm}$ in the bulk are $1.0 \pm 0.2 \mathrm{ps}$ and $1.2 \pm 0.4 \mathrm{ps}$, respectively, both of which are much shorter than those for the surface. The decay times for $660 \mathrm{~nm}$ and $745 \mathrm{~nm}$ in the bulk are $103 \pm 10 \mathrm{ps}$ and $121 \pm 9 \mathrm{ps}$, respectively, both of which are also faster than the value of $500 \pm 40$ ps for the surface. the surface states could not be fitted with a single exponential function, suggesting that there is more than one process behind the rise for the two surface states. Fittings with a double exponential function to the kinetic traces of the rise time at $660 \mathrm{~nm}$ yield a fast component of $1.8 \pm 0.2 \mathrm{ps}$ and a slow component of $25.4 \pm 3.1 \mathrm{ps}$ under the pump fluence of $1.27 \mu \mathrm{J} / \mathrm{cm}^{2}$. The two surface processes are still much slower as compared to that of $1.0 \pm 0.2 \mathrm{ps}$ in the bulk in Fig. 4(b), in which the fast rise at the early time was due to the transport of photogenerated holes to the bulk. The instrument response function (IRF) for our system depends mainly on the pulse duration of the SWIR. The pulse duration of the SWIR was measured by interfacial XFROG from a z-cut quartz single crystal and was determined to be $\sim 200 \mathrm{fs}$.

To further understand the nature of the two surface processes, the fluence of the pump was varied at different carrier densities. Figure 5(a) displays fluence-dependent kinetic traces at $745 \mathrm{~nm}$ as an example for p-type oxidized GaAs (100) under the photoexcitation of $796 \mathrm{~nm}$. The fluence-dependent kinetic traces at $660 \mathrm{~nm}$ are found in Fig. S3 of the supplementary material. The surface recombination process speeds up as the fluence is increased to $12.66 \mu \mathrm{J} / \mathrm{cm}^{2}$, suggesting that surface trapped electrons annihilate to dissipate excess energy at a longer time scale of 420 ps, as shown in Fig. 5(b). At the early time, the fast rise component tends to dominate with increasing fluence without changing the time constant with the applied fluence, while the slow rise component gradually decreases and gets shorter and shorter as the fluence is increased. The slow rise was attributed to the population of the surface states. However, the fast component at the early time might originate possibly from two mechanisms as follows. One possibility is that there exists a different surface state that has different energy barriers for the population of photo-generated electrons. The other possibility is the build-up of the surface photovoltage (SPV), which changes the depletion width towards the flat-band potential. This process is also called electric-field induced SHG (EFISH) process. ${ }^{13,15,48,91-101}$ The former was excluded since the two surface states exhibit almost the same population rate in the early time upon the photoexcitation, as displayed in Fig. 5(c). Therefore, the SPV mechanism was assigned to the fast rise in the fluence-dependent kinetics in Fig. 5(a). Electrons are generated and collected by the surface of p-type GaAs, leading to the decrease in the surface potential. The electric field in the space charge region is screened by the mobile carriers created by the optical pump pulse. As the fluence is increased, the SPV component almost governs the early rise process, as displayed in Fig. 5(c). Under a high fluence of $10.0 \mu \mathrm{J} / \mathrm{cm}^{2}$, the transient signal at the maximum tends to saturate, as shown in Fig. 5(d). This photo-saturation suggests that the applications of the sufficiently intense above-bandgap illumination to the surface result in almost complete flattening of the surface band-bending of p-type GaAs.

Figure 5(c) shows the time constants obtained for both the fast component and the slow component by fitting the early time of the fluence dependent data in Fig. 5(a) and Fig. S3 with a double exponential function. The time constants for 

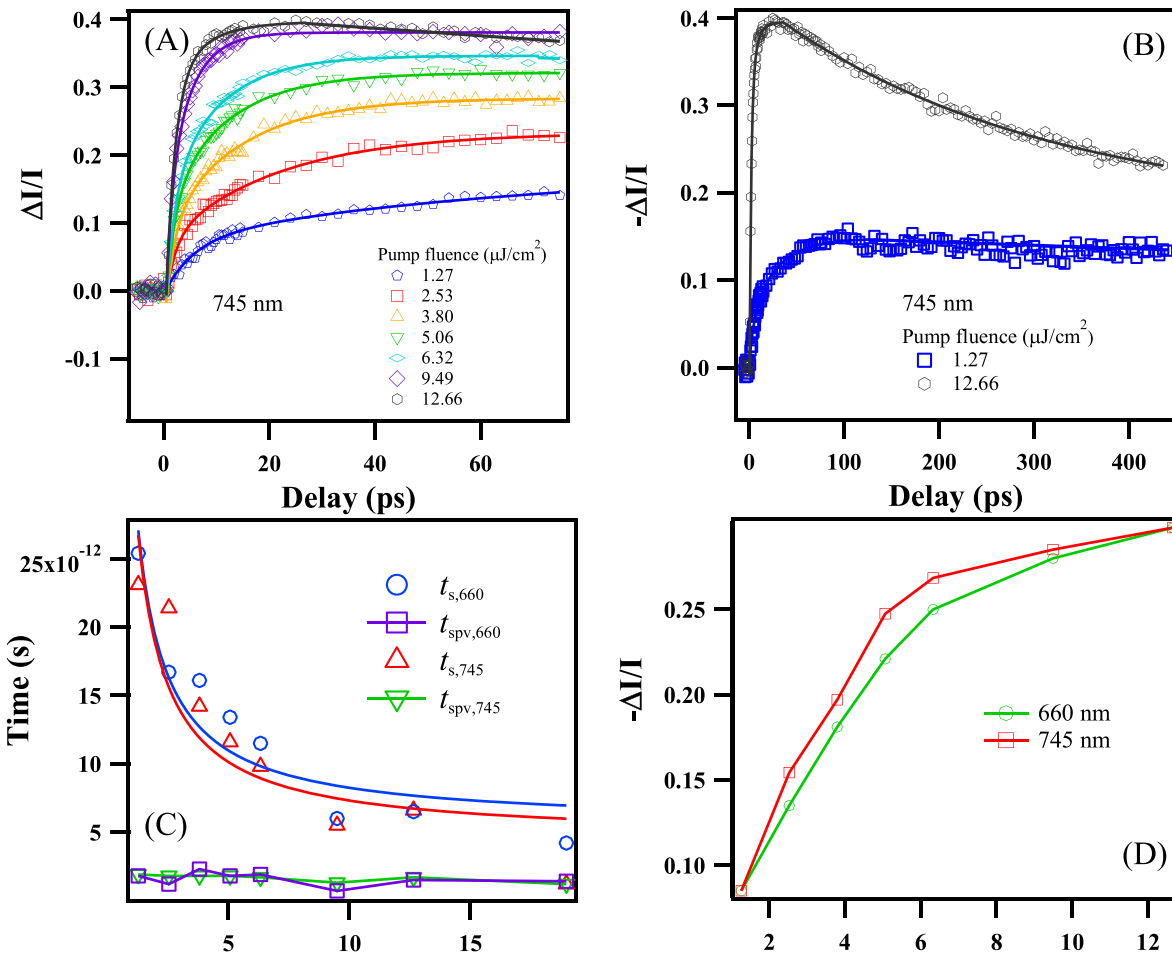

Pump Fluence $\left(\mu \mathrm{J} / \mathrm{cm}^{2}\right)$

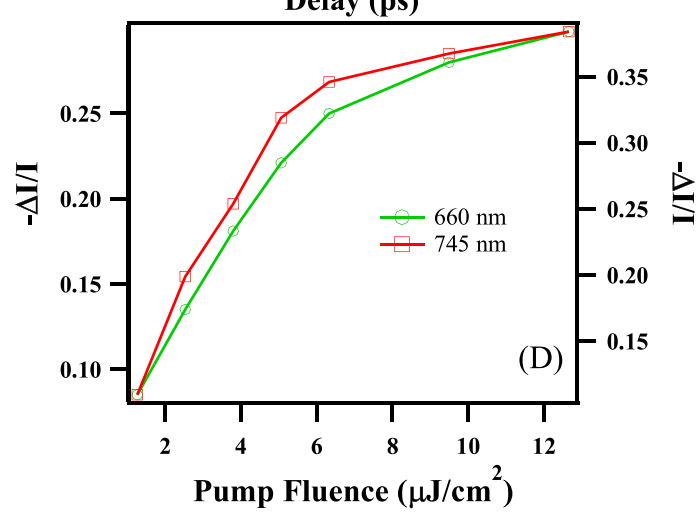

FIG. 5. (a) Fluence-dependent kinetic traces of the change in surface responses at a detection wavelength of $745 \mathrm{~nm}$ for p-type GaAs (100) under photoexcitation of $796 \mathrm{~nm}$ at a short time scale of $80 \mathrm{ps}$. Those at $660 \mathrm{~nm}$ are found in the supplementary material. (b) At a longer time scale of $420 \mathrm{ps}$, kinetic traces of the change in surface responses at a detection wavelength of $745 \mathrm{~nm}$ for a low pump fluence of $1.27 \mu \mathrm{J} / \mathrm{cm}^{2}$ (blue hexagons) and a high pump fluence of $12.66 \mu \mathrm{J} / \mathrm{cm}^{2}$ (black squares). (c) The fast rise component $\left(t_{\mathrm{spv}}\right)$ and slow rise component $\left(t_{\mathrm{s}}\right)$ at $660 \mathrm{~nm}$ and $745 \mathrm{~nm}$ plotted as a function of applied pump fluence. (d) The transient signals at the maximum for $660 \mathrm{~nm}$ (green circles) and $745 \mathrm{~nm}$ (red squares) as a function of applied pump fluence.

the fast SPV component $\left(t_{\mathrm{spv}}\right)$ remain almost constant at the different applied fluences since the build-up of the SPV is a wavelength-independent process. On the other hand, the population times of the slow surface population component $\left(t_{s}\right)$ vary with fluence. This process proceeds with the secondorder carrier population of the surface states, as mentioned earlier. Based upon the standard physical chemistry textbooks, ${ }^{102}$ a simple model for the second-order reaction was used to fit $t_{s}: t_{s}=t_{0}+1 /\left(k_{s} \times n_{0}\right)$, where $k_{s}$ is the population rate of the surfaces states and $n_{0}$ is the number density of photoexcited electrons. Surface sites accessible for native oxidized p-type GaAs were found to be $3.0 \times 10^{13} \mathrm{~cm}^{-2}$ (see the supplementary material), which is in agreement with the value reported in the literature. ${ }^{103,104}$ A fluence of $10 \mu \mathrm{J} / \mathrm{cm}^{2}$ fully populates all the surface states for $\mathrm{p}$-type oxidized GaAs, as seen in the supplementary material. Thus, the population rate constants at $660 \mathrm{~nm}$ and $745 \mathrm{~nm}$ were found to be similar with a value of $0.012( \pm 0.002) \times 10^{12} \mathrm{~s}^{-1} \mathrm{~cm}^{2}$. These results suggest that two surface states have similar energy barriers for population of photo-generated electrons.

In an effort to understand time-dependent surface responses, a kinetic model was established for electrons in the surface region and surface states as follows. According to the downward band bending in the p-type GaAs, holes are driven to the bulk region and photoexcited electrons are trapped to the surfaces, as schematically depicted in Fig. 6. Surface electrons act to disrupt equilibrium space charge region conditions by more efficiently screening the space charge field (the photo-voltage effect) and temporally changing the occupancy of the surface levels. Photo-excited electrons tend to move towards the depleted surface region with a buildup rate of SPV, $k_{\mathrm{SPV}}$. In the meantime, part of the electrons is populated in the surface states. This population proceeds with the second-order populating rate of surfaces states, $k_{s_{i}}$, in order for surface charge to remain neutral. Two independent equations for the surface carrier processes are given by

$$
\begin{aligned}
& \frac{d n_{e}(t)}{d t}=\mathrm{G}(\mathrm{t})+k_{\mathrm{SPV}} n_{e}(\mathrm{t})-k_{e} n_{e}(\mathrm{t}), \\
& \frac{d n_{\mathrm{s}_{i}}(\mathrm{t})}{d \mathrm{t}}=\mathrm{G}(\mathrm{t})+k_{\mathrm{s}_{i}} n_{\mathrm{si}}^{2}(\mathrm{t})-k_{r} n_{\mathrm{s}_{i}}(\mathrm{t}),
\end{aligned}
$$

where $G(t)$ is the instantaneous photoexcited rate of electrons related to the surfaces, $n_{e}(t)$ and $n_{s_{i}}(t)$ are the timedependent electron density in the depleted region and the 


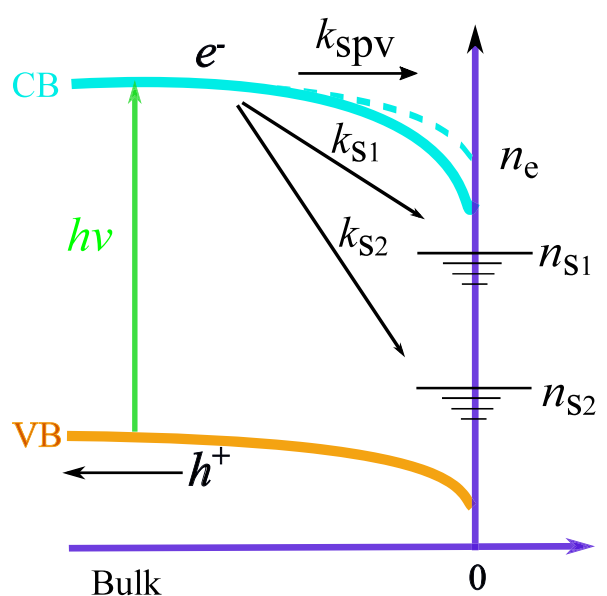

FIG. 6. Schematic of the energy diagrams of $p$-type GaAs for surface carrier relaxations upon photoexcitation. $C B$ and VB represent conduction band and valence band, respectively. $k_{S P V}$ is the buildup rate of surface photovoltage (SPV). $k_{\mathrm{si}}$ and $n_{\mathrm{si}}$ are the formation rate and number density of surfaces states $(i=1,2)$, respectively. $n_{\mathrm{e}}$ is the electron density in the depleted region.

time-dependent surface trapped electron density in the two surface states ( $i=1$ and 2$)$, respectively, $k_{\mathrm{e}}$ is the recombination rate of surface electrons, and $k_{\mathrm{r}}$ is the decay rate of the surface trapped electrons. Here, $k_{\mathrm{r}}$ can be neglected since the escape rate is so low. Surface electron-electron annihilation could occur at a longer time scale under a high fluence of $12.7 \mu \mathrm{J} / \mathrm{cm}^{2}$, as shown in Fig. 5(b). For simplicity, the annihilation is not considered here. Time-dependent changes in surface responses are the sum over both the electrons densities at the p-type GaAs surface. The electric-field induced response, namely SPV, is of non-resonant origin and independent of a detected wavelength. On the other hand, trapped electrons in the surface states are wavelength-dependent. Thus, the time-dependent change in the surface responses is given by

$$
-\frac{\Delta \mathrm{I}}{\mathrm{I}}(\lambda, \mathrm{t})=a_{1} * n_{e}(\mathrm{t})+a_{2} * n_{s_{i}}(\lambda, t)
$$

The kinetic processes at the oxidized p-type GaAs (100) surface include both the population and recombination of the surface states after photoexcitation, in addition to the buildup of the SPV in the surface region. According to the downward band bending in the p-type GaAs, the photo-excited holes are driven to the bulk region and the electrons move towards the surface region. The electrons subsequently relax by electron-hole two-body surface recombination. Photogenerated carriers act to disrupt equilibrium SCR conditions by more efficiently screening the space electric field or temporally changing the occupancy of the surface states. (1) Buildup of the SPV. The formation of the SPV occurs only if carrier generation is followed by net charge redistribution. In our case, the SPV with a rate of $0.56 \pm 0.07 \mathrm{ps}^{-1}$ is a competition step with the population of the surface states on p-type GaAs. At a high fluence above $8 \mu \mathrm{J} / \mathrm{cm}^{2}$, the SPV dominates in the early time. The SPV build-up step is the first-order process and does not change with the newly generated electronholes in the heavily doped p-type GaAs. (2) Population of the surface states. The population of the surface states is significantly slower than that for the SPV. The population rate of the surface states varies with the applied fluence, implying that the population is a two-body process. The population rate of the surface states depends on the electron capture cross section and the carrier thermal velocity at the surfaces. The population rate constants for the two surface states are similar, which is on the order of $0.012( \pm 0.002) \times 10^{12} \mathrm{~s}^{-1} \mathrm{~cm}^{2}$. Surface states accessible for native oxidized $\mathrm{p}$-type GaAs are estimated to be about $3 \times 10^{13} \mathrm{~cm}^{-2}$, which is in agreement with our experimental results (see the supplementary material). ${ }^{7,105}$ As such, the surface states are fully populated even with a pump fluence above $10.0 \mu \mathrm{J} / \mathrm{cm}^{2}$. (3) Surface recombination: the photoexcitation creates electron-hole pairs near the surface, which recombine either directly or indirectly through the surface states. The surface electron-hole recombination has a rate of $0.002 \pm 0.0002 \mathrm{ps}^{-1}$ for the oxidized p-type GaAs (100). At a higher fluence above $5 \mu \mathrm{J} / \mathrm{cm}^{2}$, the indirect recombination of surface-trapped electronelectron annihilation becomes prominent in the relaxation process, leading to a faster relaxation of hot surface trapped electrons.

\section{SUMMARY AND CONCLUSIONS}

We have established a new ultrafast surface/interfacespecific spectrometer for broadband electronic SFG and SHG. The broadband surface/interface spectrometer was based upon a stable two-stage BIBO crystal-based optical parametric amplifier, which generates a strong broadband short-wave IR from $1200 \mathrm{~nm}$ to $2200 \mathrm{~nm}$ and is readily extended down to $600 \mathrm{~nm}$. A resultant surface spectrum covers almost all visible light from $480 \mathrm{~nm}$ to $760 \mathrm{~nm}$, combined with a broadband second harmonic generation of the short-wave IR. The temporal pulse of the broad short-wave IR was as short as $200 \mathrm{fs}$. The output power was as high as $250 \mu \mathrm{J}$ with a $796 \mathrm{~nm}$ fundamental light of $1.5 \mathrm{~mJ}$ per pulse. These unique features for the broadband laser source enable us to develop both steady-state and transient electronic surface/interface-specific techniques. These ultrafast interface/surface-specific tools are anticipated to apply to more topics related to environmental issues, materials science, and biological systems.

Based on the newly established broadband OPA, we have further developed the steady-state and transient ESFG and ESHG techniques to investigate the surface structure and dynamics of charges at oxidized p-type GaAs (100) semiconductor surfaces. Both the steady-state and transient experiments have shown that two surface states exist inside the bandgap of the GaAs. The kinetic processes at the GaAs surface include both the population and recombination of the surface states after photoexcitation, in addition to the build-up of the SPV. The SPV competes with the population of the surface states on the p-type GaAs surface. The SPV dominates in the early time, with the first-order rate of 
$0.56 \pm 0.07 \mathrm{ps}^{-1}$, while the population of the surface states exhibits a two-body behavior when the photo-generated electrons tend to saturate the surface states. The second order populating rate constants for the two surface states are similar, which are $0.012( \pm 0.002) \times 10^{12} \mathrm{~s}^{-1} \mathrm{~cm}^{2}$. The photogenerated electron-hole pairs near the surface recombine both directly and indirectly through the surface states. The direct recombination occurs at a time constant of $0.002 \pm$ $0.0002 \mathrm{ps}^{-1}$ for the oxidized p-type GaAs (100). The indirect annihilation recombination becomes dominant once the surface states were fully occupied.

The newly developed transient surface/interface-specific electronic spectroscopies provide the structure and dynamics of interfacial/surface states related to environmental issues, materials science, and biological systems. These methodologies are readily applied to not only classical inorganic semiconductor surfaces and their interfaces with other materials but also organic semiconductor surfaces, buried interfaces, and hybrid interfaces.

\section{SUPPLEMENTARY MATERIAL}

Calculations of coherent length of SFG from the GaAs surface and photo-generated electron density are shown in the supplementary material. Transient two-dimensional pseudocolor SHG spectra from the p-type oxidized GaAs (100) surface, transient SHG spectra of the GaAs surface at different delay times, and fluence-dependent kinetic traces of the change in surface responses at a detection wavelength of $660 \mathrm{~nm}$ for p-type GaAs (100) under photoexcitation of $796 \mathrm{~nm}$ are given in Figs. S1-S3 of the supplementary material, respectively.

\section{ACKNOWLEDGMENTS}

Y.R. acknowledges support from Utah State University. Y.R. values with full respect that this work was dedicated to Dr. Kenneth B. Eisenthal and Dr. Nicholas J. Turro for their mentor-ship, advisor-ship, and professionalism. The authors are thankful to Dr. Pancho Tzankov, Dr. Zhiqiang Li, Dr. Dezheng Sun, and Dr. Bolei Xu for their beneficial discussions and suggestions.

\section{REFERENCES}

${ }^{1}$ H. Lüth, Solid Surfaces, Interfaces and Thin Films (Springer, 2001), Vol. 4.

${ }^{2}$ W. Mönch, Semiconductor Surfaces and Interfaces (Springer Science \& Business Media, 2013), Vol. 26.

${ }^{3}$ Y. Peter, and M. Cardona, Fundamentals of Semiconductors: Physics and Materials Properties (Springer Science \& Business Media, 2010)

${ }^{4}$ A. Many, Y. Goldstein, and N. B. Grover, Semiconductor Surfaces (NorthHolland Publishing Co. (u.a.), 1971).

${ }^{5}$ V. Heine, Phys. Rev. 138, A1689 (1965).

${ }^{6}$ Z. Zhang and J. T. Yates, Jr., Chem. Rev. 112, 5520 (2012).

${ }^{7}$ T. Dekorsy, T. Pfeifer, W. Kütt, and H. Kurz, Phys. Rev. B 47, 3842 (1993).

${ }^{8}$ M. F. Crommie, C. P. Lutz, and D. M. Eigler, Science 262, 218 (1993).

${ }^{9}$ R. M. Feenstra, Phys. Rev. B 50, 4561 (1994).

${ }^{10}$ E. Knoesel, A. Hotzel, and M. Wolf, J. Electron Spectrosc. Relat. Phenom. 88, 577 (1998).
${ }^{11}$ S. Tanaka and K. Tanimura, Surf. Sci. 529, L251 (2003).

${ }^{12}$ K. Prabhakaran and T. Ogino, Surf. Sci. 325, 263 (1995).

${ }^{13}$ H. Park, M. Gutierrez, X. Wu, W. Kim, and X.-Y. Zhu, J. Phys. Chem. C 117, 10974 (2013).

${ }^{14}$ C. Nelson, J. Luo, A.-Y. Jen, R. Laghumavarapu, D. Huffaker, and X.-Y. Zhu, J. Phys. Chem. C 118, 27981 (2014).

${ }^{15}$ W. A. Tisdale, K. J. Williams, B. A. Timp, D. J. Norris, E. S. Aydil, and X.-Y. Zhu, Science 328, 1543 (2010).

${ }^{16}$ A. J. Goodman, N. S. Dahod, and W. A. Tisdale, J. Phys. Chem. Lett. 9, 4227 (2018).

17J. P. Long, H. R. Sadeghi, J. C. Rife, and M. N. Kabler, Phys. Rev. Lett. 64, 1158 (1990)

${ }^{18}$ N. J. Halas and J. Bokor, Phys. Rev. Lett. 62, 1679 (1989).

${ }^{19}$ M. W. Rowe, H. Liu, G. P. Williams, and R. T. Williams, Phys. Rev. B 47, 2048 (1993).

${ }^{20}$ P. Siffalovic, M. Drescher, and U. Heinzmann, Europhys. Lett. 60, 924 (2002).

${ }^{21}$ Y. R. Shen, Nature 337, 519 (1989).

${ }^{22}$ P. B. Miranda and Y. R. Shen, J. Phys. Chem. B 103, 3292 (1999).

${ }^{23}$ K. B. Eisenthal, Annu. Rev. Phys. Chem. 43, 627 (1992).

${ }^{24}$ K. B. Eisenthal, Chem. Rev. 96, 1343 (1996).

${ }^{25}$ G. L. Richmond, Chem. Rev. 102, 2693 (2002).

${ }^{26}$ H. F. Wang, W. Gan, R. Lu, Y. Rao, and B. H. Wu, Int. Rev. Phys. Chem. 24, 191 (2005).

${ }^{27}$ F. M. Geiger, Annu. Rev. Phys. Chem. 60, 61 (2009).

${ }^{28}$ Z. Chen, Y. Shen, and G. A. Somorjai, Annu. Rev. Phys. Chem. 53, 437 (2002).

${ }^{29}$ A. M. Jubb, W. Hua, and H. C. Allen, Annu. Rev. Phys. Chem. 63, 107 (2012).

${ }^{30} \mathrm{H}$. Arnolds and M. Bonn, Surf. Sci. Rep. 65, 45 (2010).

${ }^{31}$ C. Y. Wang, H. Groenzin, and M. J. Shultz, J. Phys. Chem. B 108, 265 (2004).

${ }^{32}$ C. M. Johnson and S. Baldelli, Chem. Rev. 114, 8416 (2014).

${ }^{33}$ B. J. Berne, J. T. Fourkas, R. A. Walker, and J. D. Weeks, Acc. Chem. Res. 49, 1605 (2016).

${ }^{34}$ K. Y. Kung, P. Chen, F. Wei, Y. R. Shen, and G. A. Somorjai, Surf. Sci. 463, L627 (2000).

${ }^{35}$ M. Makarem, C. M. Lee, D. Sawada, H. M. O’Neill, and S. H. Kim, J. Phys. Chem. Lett. 9, 70 (2017).

${ }^{36}$ H. Vanselous, A. M. Stingel, and P. B. Petersen, J. Phys. Chem. Lett. 8, 825 (2017).

${ }^{37}$ H. Y. Wang, T. Gao, and W. Xiong, ACS Photonics 4, 1839 (2017).

${ }^{38}$ A. P. Moon, R. Pandey, J. A. Bender, D. E. Cotton, B. A. Renard, and S. T. Roberts, J. Phys. Chem. C 121, 18653 (2017).

${ }^{39}$ J. E. Laaser and M. T. Zanni, J. Phys. Chem. A 117, 5875 (2013).

${ }^{40}$ E. C. Y. Yan, L. Fu, Z. G. Wang, and W. Liu, Chem. Rev. 114, 8471 (2014).

${ }^{41}$ J. F. Liljeblad and E. Tyrode, J. Phys. Chem. C 116, 22893 (2012).

${ }^{42}$ I. V. Stiopkin, C. Weeraman, P. A. Pieniazek, F. Y. Shalhout, J. L. Skinner, and A. V. Benderskii, Nature 474, 192 (2011).

${ }^{43}$ A. U. Chowdhury, F. Liu, B. R. Watson, R. Ashkar, J. Katsaras, C. Patrick Collier, D. A. Lutterman, Y.-Z. Ma, T. R. Calhoun, and B. Doughty, Opt. Lett. 43, 2038 (2018).

${ }^{44}$ R. R. Kumal, H. Nguyenhuu, J. E. Winter, R. L. McCarley, and L. H. Haber, J. Phys. Chem. C 121, 15851 (2017).

${ }^{45}$ D. Elsenbeck, S. K. Das, and L. Velarde, Phys. Chem. Chem. Phys. 19, 18519 (2017).

${ }^{46}$ N. G. Rey and D. D. Dlott, J. Electroanal. Chem. 800, 114 (2017).

${ }^{47}$ T. C. Anglin and J. C. Conboy, Biochemistry 48, 10220 (2009).

${ }^{48}$ Y.-C. Wen, S. Zha, X. Liu, S. Yang, P. Guo, G. Shi, H. Fang, Y. R. Shen, and C. Tian, Phys. Rev. Lett. 116, 016101 (2016).

${ }^{49}$ G. H. Deng, X. Li, S. L. Liu, Z. Zhang, Z. Lu, and Y. Guo, J. Phys. Chem. C 120, 12032 (2016).

${ }^{50}$ Y. Ma, Y. Xie, L. Lin, L. Zhang, M. Liu, Y. Guo, Z. Lan, and Z. Lu, J. Phys. Chem. C 121, 23541 (2017).

${ }^{51}$ R.-J. Feng, L. Lin, Y.-Y. Li, M.-H. Liu, Y. Guo, and Z. Zhang, Biophys. J. 112, 2173 (2017). 
${ }^{52}$ W. Wu, H. Fang, F. Yang, S. Chen, X. Zhu, Q. Yuan, and W. Gan, J. Phys. Chem. C 120, 6515 (2016).

${ }^{53}$ S. Liu, A.-a. Liu, B. Wen, R. Zhang, C. Zhou, L.-M. Liu, and Z. Ren, J. Phys. Chem. Lett. 6, 3327 (2015).

${ }^{54}$ S. Nihonyanagi, S. Yamaguchi, and T. Tahara, Chem. Rev. 117, 10665 (2017).

${ }^{55}$ J. Sung, Y. Jeon, D. Kim, T. Iwahashi, K. Seki, T. Iimori, and Y. Ouchi, Colloids Surf, A 284, 84 (2006).

${ }^{56} \mathrm{~S}$. Ye, F. Wei, H. Li, K. Tian, and Y. Luo, Advances in Protein Chemistry and Structural Biology (Elsevier, 2013), pp. 213.

${ }^{57}$ X. Shang, K. Nguyen, Y. Rao, and K. B. Eisenthal, J. Phys. Chem. C 112, 20375 (2008).

${ }^{58}$ Y. Ma, J. Hou, W. Hao, J. Liu, L. Meng, and Z. Lu, Phys. Chem. Chem. Phys. 20, 17199 (2018)

${ }^{59}$ T. Ishiyama and A. Morita, Annu. Rev. Phys. Chem. 68, 355 (2017).

${ }^{60}$ Y. Cao, S. Chen, Y. Li, Y. Gao, D. Yang, Y. R. Shen, and W.-T. Liu, Sci. Adv. 2, e1601162 (2016).

${ }^{61}$ D. Lis, E. H. G. Backus, J. Hunger, S. H. Parekh, and M. Bonn, Science 344, 1138 (2014).

${ }^{62}$ Y. Rao, D. Song, N. J. Turro, and K. B. Eisenthal, J. Phys. Chem. C 112, 13572 (2008).

${ }^{63}$ J. A. Carter, Z. Wang, and D. D. Dlott, Acc. Chem. Res. 42, 1343 (2009).

${ }^{64} \mathrm{Z}$. Li, J. Wang, Y. Li, and W. Xiong, J. Phys. Chem. C 120, 20239 (2016).

${ }^{65}$ Y. Rao, S.-Y. Hong, N. J. Turro, and K. B. Eisenthal, J. Phys. Chem. C 115, 11678 (2011).

${ }^{66}$ L. J. Richter, T. P. Petralli-Mallow, and J. C. Stephenson, Opt. Lett. 23, 1594 (1998).

${ }^{67}$ L. Velarde, X. Y. Zhang, Z. Lu, A. G. Joly, Z. M. Wang, and H. F. Wang, J. Chem. Phys. 135, 241102 (2011).

${ }^{68}$ H. F. Wang, E. Borguet, and K. B. Eisenthal, J. Phys. Chem. A 101, 713 (1997).

${ }^{69}$ T. F. Heinz, C. K. Chen, D. Ricard, and Y. R. Shen, Phys. Rev. Lett. 48, 478 (1982).

${ }^{70}$ W. H. Steel and R. A. Walker, Nature 424, 296 (2003).

${ }^{71}$ Y. M. Li, J. M. Wang, and W. Xiong, J. Phys. Chem. C 119, 28083 (2015).

${ }^{72}$ Y. Rao, M. Xu, S. Jockusch, N. J. Turro, and K. B. Eisenthal, Chem. Phys. Lett. 544, 1 (2012)

${ }^{73}$ B. Doughty, Y.-Z. Ma, and R. W. Shaw, J. Phys. Chem. C 119, 2752 (2015).

${ }^{74}$ S. Yamaguchi and T. Tahara, J. Phys. Chem. B 108, 19079 (2004).

${ }^{75}$ V. Petrov, M. Ghotbi, O. Kokabee, A. Esteban-Martin, F. Noack, A. Gaydardzhiev, I. Nikolov, P. Tzankov, I. Buchvarov, and K. Miyata, Laser Photonics Rev. 4, 53 (2010).

${ }^{76}$ V. Petrov, A. Gaydardzhiev, I. Nikolov, I. Buchvarov, P. Tzankov, and F. Noack, in Nonlinear Frequency Generation and Conversion: Materials, Devices, and Applications VII (International Society for Optics and Photonics, 2008), p. 68750X.
${ }^{77}$ M. Ghotbi, M. Ebrahim-Zadeh, V. Petrov, P. Tzankov, and F. Noack, Opt. Express 14, 10621 (2006).

${ }^{78}$ Y. Rao, N. J. Turro, and K. B. Eisenthal, J. Phys. Chem. C 114, 17703 (2010).

${ }^{79}$ B. Xu, Y. Wu, D. Sun, H.-L. Dai, and Y. Rao, Opt. Lett. 40, 4472 (2015).

${ }^{80}$ S. Marc, G. Avishek, B. Jens, Y. Susumu, M. Michiel, and B. Mischa, New J. Phys. 9, 390 (2007).

${ }^{81}$ K. Sekiguchi, S. Yamaguchi, and T. Tahara, J. Chem. Phys. 128, 114715 (2008).

${ }^{82}$ E. H. G. Backus, J. D. Cyran, M. Grechko, Y. Nagata, and M. Bonn, J. Phys. Chem. C 122, 2401 (2018).

${ }^{83}$ Z. Zhang, J. Kim, R. Khoury, M. Saghayezhian, L. H. Haber, and E. Plummer, Surf. Sci. 664, 21 (2017).

${ }^{84} \mathrm{C}$. Yamada and T. Kimura, Phys. Rev. Lett. 70, 2344 (1993).

${ }^{85} \mathrm{C}$. Yamada and T. Kimura, Phys. Rev. B 49, 14372 (1994).

${ }^{86}$ S. Janz and Z. H. Lu, J. Opt. Soc. Am. B 14, 1647 (1997).

${ }^{87}$ T. A. Germer, K. W. Kołasin-acuteski, J. C. Stephenson, and L. J. Richter, Phys. Rev. B 55, 10694 (1997).

${ }^{88}$ W. E. Spicer, I. Lindau, P. Skeath, and C. Y. Su, J. Vac. Sci. Technol. 17, 1019 (1980).

${ }^{89}$ W. E. Spicer, Z. Liliental-Weber, E. Weber, N. Newman, T. Kendelewicz, R. Cao, C. McCants, P. Mahowald, K. Miyano, and I. Lindau, J. Vac. Sci. Technol., B: Microelectron. Nanometer Struct. 6, 1245 (1988).

${ }^{90}$ D. E. Aspnes, Surf. Sci. 132, 406 (1983)

${ }^{91}$ C. H. Lee, R. K. Chang, and N. Bloembergen, Phys. Rev. Lett. 18, 167 (1967).

${ }^{92}$ B. F. Levine and C. G. Bethea, J. Chem. Phys. 63, 2666 (1975).

${ }^{93} \mathrm{~J}$. Qi, M. S. Yeganeh, I. Koltover, A. G. Yodh, and W. M. Theis, Phys. Rev. Lett. 71, 633 (1993).

${ }^{94}$ X. Zhao, S. Ong, H. Wang, and K. B. Eisenthal, Chem. Phys. Lett. 214, 203 (1993).

${ }^{95}$ A. Md Shafiul, D. Akemi, and M. G.-D. Julianne, J. Phys.: Condens. Matter 26, 244107 (2014)

${ }^{96}$ D. S. Jordan, C. J. Hull, J. M. Troiano, S. C. Riha, A. B. F. Martinson, K. M. Rosso, and F. M. Geiger, J. Phys. Chem. C 117, 4040 (2013).

${ }^{97}$ G. Gonella, C. Lütgebaucks, A. G. F. de Beer, and S. Roke, J. Phys. Chem. C 120, 9165 (2016).

${ }^{98}$ P. E. Ohno, S. A. Saslow, H.-f. Wang, F. M. Geiger, and K. B. Eisenthal, Nat. Commun. 7, 13587 (2016).

${ }^{99}$ S. Ong, X. Zhao, and K. B. Eisenthal, Chem. Phys. Lett. 191, 327 (1992).

${ }^{100}$ T. Joutsuka and A. Morita, J. Phys. Chem. C 122, 11407 (2018).

${ }^{101}$ K. C. Jena, P. A. Covert, and D. K. Hore, J. Phys. Chem. Lett. 2, 1056 (2011).

${ }^{102}$ P. Atkins and J. d. Paula, Atkins' Physical Chemistry (Oxford University Press, Oxford, 2002).

${ }^{103}$ E. W. Kreutz, Phys. Status Solidi A 56, 687 (1979).

${ }^{104}$ G. S. Chang, W. C. Hwang, Y. C. Wang, Z. P. Yang, and J. S. Hwang, J. Appl. Phys. 86, 1765 (1999).

${ }^{105}$ C. J. Spindt and W. E. Spicer, Appl. Phys. Lett. 55, 1653 (1989). 